\title{
El patrimonio cultural y natural como recurso didáctico en la práctica docente mayangna, panamahka
}

\author{
The cultural and natural heritage as a teaching resource \\ in the Mayangna teaching practice, panamahka
}

Kevin González Hodgson'

\section{Resumen}

El siguiente documento corresponde a una experiencia docente y da cuenta sobre los elementos fundamentales del dossier didáctico Patrimonio Cultural y Natural de la Costa Caribe de Nicaragua el cual se inserta en el marco del proyecto Generando Capacidades en Recursos Humanos Docentes y Materiales Didácticos para el Fortalecimiento de la Educación Intercultural Bilingüe (EIB); el punto de partida del dossier elaborado tuvo como propósito apoyar la labor educativa de los docentes comunitarios del territorio Mayangna Sauni As y Sauni Arungka, en la Región Autónoma de la Costa Caribe Norte, por lo que los resultados de este material didáctico sirvieron de insumo para la adecuación de planes de estudio tanto en lengua vernácula como en la modalidad castellana en efecto, como eje transversal que deberá articular el currículum escolar, particularmente en la enseñanza primaria del Sistema Educativo Autonómico Regional del país.

Palabras clave: Costa Caribe de Nicaragua, currículo, dossier didáctico, Educación Intercultural Bilingüe, patrimonio cultural y natural.

\section{Abstract}

The following document corresponds to a teaching experience and reports on the fundamental elements of the Cultural and Natural Heritage of the Caribbean Coast of Nicaragua didactic dossier which is inserted in the framework of the Generating Capacities in Human Teaching Resources and Didactic Materials for the Strengthening of Intercultural Bilingual Education (IBE) Project; The starting point of the dossier was intended to support the educational work of the community teachers of the Mayangna Sauni As and Sauni Arungka territory, in the Autonomous Regions of the North Caribbean Coast, consequently the results of this didactic material served as input for the adequacy of curricula in both the vernacular language and the Castilian modality in effect, as a transversal axis that should articulate the school curriculum, particularly in the primary education of the Regional Autonomous Educational System of the country.

Key words: Caribbean Coast of Nicaragua, curriculum, cultural and natural heritage, educational dossier, intercultural bilingual education

\footnotetext{
1 Máster en métodos de investigación científica. Arqueólogo asociado al Centro Arqueológico de Documentación e Investigación de la Universidad Nacional Autónoma de Nicaragua-Managua Correo: hodgsonk27@hotmail.com ORCID: https://orcid.org/0000-0003-1127-4309
}

Recibido: 02/10/2018 - Aprobado: 22/01/2019 


\section{Introducción}

El presente artículo da cuenta sobre la experiencia de algunos años impartiendo el módulo didáctico Patrimonio Cultural y Natural de la Costa Caribe de Nicaragua, cuyo punto de partida en esta ocasión se insertó en el proyecto macro: Generando Capacidades en Recursos Humanos Docentes y Materiales Didácticos para el Fortalecimiento de la Educación Intercultural Bilingüe (IEB) ${ }^{2}$, en los territorios Mayangna Sauni As y Sauni Arungka o Matumbak en la RACCN33 y ejecutado con la asistencia técnica de la Facultad de Humanidades y Ciencias Jurídicas de la Universidad Nacional Autónoma de Nicaragua (UNAN-Managua) ${ }^{4} \mathrm{y}$ auspiciado con fondos de la Sociedad Civil en Nicaragua (FASOC).

El módulo en cuestión formó parte del plan de estudio del diplomado Diseño Curricular y Materiales Didácticos para el Fortalecimiento de la Educación Intercultural Bilingüe, el cual surge de una necesidad sentida de parte de los docentes mayangnas en los territorios señalados quienes manifestaron en todo momento inquietud y disposición por conocer e incorporar fundamentos teóricos-metodológicos y didácticos no sólo para los alcances propuestos en el proyecto sino también, afianzar conocimientos que les permitan enfrentar los nuevos desafíos que demandan los escenarios educativos en el presente. El objetivo central del diplomado fue contribuir al fortalecimiento de la Educación Intercultural Bilingüe en el marco del sistema Educativo Autonómico Regional (SEAR) a través, del proceso de adecuación curricular y elaboración de materiales didácticos de los niveles educativos preescolar comunitario y formal; además, de primaria regular y multigrado; en efecto, la propuesta didáctica responde a un enfoque participativo en las modalidades señaladas por lo que se realizó una capacitación de corta duración ${ }^{5}$ focalizado con 35 docentes y con la participación de 6 asesores pedagógicos del MINED ${ }^{6}$ de Rosita y Bonanza con niveles académicos heterogéneos.

Como parte de las transformaciones curriculares sistemáticas que se realizan desde el sistema nacional de educación y que su propósito es mejorar la calidad de los procesos de enseñanza-aprendizaje se sustentan en el caso de la Costa Caribe por el Sistema Educativo Autonómico Regional, que recoge importantes ejes conceptuales como parte de su política entre ellos autonomía categoría esencial para el ejercicio de la interculturalidad como parte del proceso permanente de construcción, establecimiento y fortalecimiento de espacios de diálogo comunicación e interacción entre personas, comunidades y pueblos de diferentes culturas (Álvarez, 2017, p. 87), interculturalidad, equidad, solidaridad, pertinencia y calidad que de manera práctica implica el reconocimiento de la diversidad cultural y multiétnica, la sustentación de la capacidad de intercambio, enriquecimiento mutuo entre las distintas experiencias latinoamericanas de Educación Intercultural Bilingüe, además, de información, conocimientos, valores morales y promueve prácticas de culturas propias y de otras que apunten hacia formas de ayuda mutua y búsqueda del bien común.

Justamente, un eje importante en el contexto globalizante en el que vive el mundo es el de promover la cultura nacional que en el caso de la Constitución Política de Nicaragua en el Artículo 126 en su título VII Educación y Cultura establece que: Es deber del Estado promover el rescate, desarrollo y fortalecimiento de la cultura nacional sustentada en la participación creativa del pueblo pero además, el mismo Estado apoyará la cultura nacional en todas sus expresiones, sean de carácter colectivo o de creadores individuales. Adicionalmente, el patrimonio cultural y natural de la Costa Caribe es una de las manifestaciones fundamentales de esta región del país y como tal debe ser incluido en las enseñanzas de los distintos niveles educativos, no como asignatura ni como tema aparte, sino como eje transversal que articula el currículum nacional y regional.

\footnotetext{
2 Previamente, al diplomado se realizó un taller de inducción para luego finalizar con la asesoría técnica-metodológica para la elaboración de los textos escolares como recurso didáctico para facilitar la labor educativa de los docentes comunitarios.

3 Región Autónoma de la Costa Caribe Norte de Nicaragua.

4 La coordinación central del proyecto estuvo a cargo de la Dra. Jilma Romero, la maestra Sandra Tenorio al igual que Carlos Lenys y Edgar Salazar como contraparte del Ministerio de Educación.

5 Las sesiones de trabajo se efectuaron los días 20, 21 y 22 de octubre de 2017.

6 Ministerio de Educación de Nicaragua.
} 
En efecto, la Costa Caribe es una región en el que la diversidad representa uno de los rasgos de su identidad que se expresa en la complejidad de características personales, de culturas, de etnias, de zonas geográficas, de sectores sociales, paisajes y otros que el currículo en Educación Intercultural Bilingüe debe de tomar en cuenta, es decir, atendiendo las diferencias pero sin establecer discriminaciones ni separaciones; esos principios y orientaciones curriculares tienen su razón en el SEAR y consignados además, en el Estatuto de Autonomía de las Regiones Autónomas de la Costa Caribe Nicaragüense o "Ley 28" de 1987 y del decreto "Ley de Uso Oficial de las Lenguas de las Comunidades de la Costa Caribe de Nicaragua", publicada en la Gaceta, Diario Oficial, No.183 del 27 de septiembre de 1996, que en el caso de esta última en su Artículo 2 estable que: las comunidades de la Costa Atlántica tienen derecho a sus lenguas. Es decir, reconocer la importancia de la lengua vernácula en la educación preescolar y primaria como elemento modelador de identidad el Estado está en la obligación de establecer programas especiales para el ejercicio y preservación de estos.

Cuando hablamos de eje transversal el módulo didáctico lo plantea desde la perspectiva de la educación en valores que son considerados esenciales en la búsqueda de una sociedad más humana, justa y solidaria; en ese sentido, la apreciación del patrimonio de la Costa Caribe y el compromiso personal en su defensa, conservación y mejora, es una realidad latente en el presente y por tanto deben ser acogidas en los planes de estudio donde permitan conocer las creencias, actitudes y los valores de nuestras tradiciones, valorarlos críticamente y elegir aquellas opciones que mejor favorezcan su desarrollo integral como personas; de modo que el apartado de educación busca un proceso de concienciación escolar y de conocimiento de los principales recursos del patrimonio caribeño.

\section{Materiales y métodos}

La ruta metodológica utilizada fue la de aprender-haciendo que se sintetiza en principio en la exposición teórica del docente y la participación de los educandos en estrategias como lluvias de ideas y puesta en común, actividades de prácticas grupales y puesta en común, investigación documental sistemática que permitiera insumos para la redacción de los textos escolares de acuerdo con las áreas de conocimiento y finalmente lectura del documento base fascículo. Tanto el facilitador como los educandos hicieron uso de medios como láminas Power Point, pizarra, papelones, marcadores de tinta indeleble, recurso audiovisual y otros medios didácticos que permitieron que los educandos expresaran sus puntos de vistas sobre el patrimonio así, como debatir en torno a las normativas de regulación, protección y preservación de los bienes patrimoniales existente en sus localidades.

\section{El dossier didáctico}

Para enfrentar el desafío de adecuación curricular y contribuir efectivamente en la práctica docente del mayangna panamahka, se estructuró un dossier que permitiera mitigar algunas deficiencias de aprendizaje, específicamente en el abordaje del tema patrimonial local permitiendo al personal en capacitación desarrollar habilidades y destrezas, capaz de comprender e interpretar procesos históricos amplios; en ese sentido, el objetivo central del dossier era:

Fortalecer la labor educativa de los docentes mayangna, panamahka en los fundamentos básicos del patrimonio natural y cultural para su puesta en práctica en los planes de estudio de los niveles preescolar comunitario y formal, primaria regular y multigrado con un enfoque holístico y sistémico capaz de distinguir y valorar las raíces propias que caracteriza a los territorios Mayangna Sauni As y Sauni Arungka, RACCN.

\section{El documento didáctico contempló 4 unidades de aprendizaje interrelacionadas:}

1) Conceptos generales sobre el patrimonio. 
2) El patrimonio natural y cultural para las localidades.

3) Gestión del patrimonio natural y cultural para las localidades.

4) Derechos de los pueblos indígenas en el marco jurídico internacional y nacional.

Se partió de los referentes conceptuales tanto del patrimonio natural y cultural enfatizando en los elementos y categorías que la conforman y su clasificación en términos generales lo cual facilitaría la lectura de las unidades posteriores; luego, se proyectó la importancia de ambos patrimonios para las localidades y su vínculo entre el patrimonio de una comunidad y sus oportunidades de desarrollo, mediante actividades como el turismo alternativo. Asimismo, se abordó las generalidades sobre la gestión del patrimonio concretando la gestión participativa de los comunitarios y el debate sobre la necesidad de proteger los recursos patrimoniales a nivel local y regional. Un último, lineamiento temático facilitó los conocimientos básicos en torno a las legislaciones vigentes para la protección y conservación del patrimonio de forma holística, así, como aquellas instituciones relacionadas a su protección, aunque por razones de tiempo del módulo se abordaron de manera somera. Hay que mencionar, además, que el dossier se estructuró en 60 páginas, con al menos una treintena de fuentes documentales y de recursos disponibles en la web (por ejemplo, entre las fuentes escritas se consultaron libros, tesis e informes arqueológicos e históricos tanto de autores nacionales como extranjeros que refieren aspectos culturales y naturales del pueblo mayangna entre ellos: Edward Conzemius, Gotz Von Houwald, Luis Hurtado de Mendoza, Jaime Incer y otros), asimismo, 1 mapa, un importante número de elementos infográficos (17 esquemas, 19 cuadros, 6 tablas y 24 fotografías), 1 glosario terminológico, la orientación bibliográfica para los capacitandos junto a un total de 25 actividades de aprendizaje que se desprenden de la información que contiene el dossier, en donde cada actividad de ejercitación tiene enunciación de contenidos y procedimientos de trabajo que tienen por finalidad profundizar y retroalimentar las temáticas de cada unidad.

Figura 1. Íconos didácticos integrados en el dossier.

Metodológicamente, los contenidos se enfocaron desde una perspectiva holística expresado en su idioma, conocimiento ancestral y expresión artística como eje fundamental de los derechos humanos, sociales, económicos y culturales del pueblo mayangna; el dossier estuvo centrado en las actividades y la ejercitación complementaria orientada en el documento más que en el contenido, por lo que para su debida comprensión y utilización pertinente debieron realizarse con disciplina por parte de los capacitados. Ahora bien, para que el dossier cumpliera un rol importante con los objetivos fijados en el diplomado fue necesario establecer cinco equipos de trabajo de acuerdo con áreas de conocimiento ${ }^{7}$ lo que hizo comprensible los contenidos programáticos.

\section{De las unidades temáticas en el plan analítico}

\section{Unidad I: Conceptos generales sobre el patrimonio}

\section{A. Objetivos}

a) Introducir a los participantes en los conceptos generales sobre el patrimonio natural y cultural desde un enfoque holístico y sistémico.

b) Abordar los bienes del patrimonio natural y cultural destacando aquellos elementos que interactúan en sus valores tangibles e intangibles, como expresión de las múltiples formas de ocupar, apropiar y explotar los territorios.

c) Precisar el concepto de diversidad biológica o biodiversidad.

$\overline{7}$ Las áreas son Preescolar, Persona, Cultura y Naturaleza, Lengua y Literatura, Matemática y Arte, Recreación y Educación Física. 
d) Valorar el papel de las reservas de biosfera como valor patrimonial de la humanidad.

\section{B. Contenido conceptual, procedimental y actitudinal}

a) Conceptos básicos sobre el patrimonio: definición y su clasificación.

b) Diversidad biológica o biodiversidad.

c) Las áreas naturales protegidas por el Estado: el caso de la Reserva de Biosfera Bosawás.

d) Patrimonio integral.

e) Entiende la definición del patrimonio y sus características.

f) Reconoce la importancia de la diversidad biológica o biodiversidad a través, de la comprensión del hecho natural de los bienes conservados para las futuras generaciones.

g) Identifica las áreas naturales protegidas por el Estado, su importancia y clasificación.

h) Maneja técnicamente las categorías que conforman el patrimonio de manera holística y sistémica.

i) Desarrolla la habilidad de análisis e interpretación de cada uno de los tipos y niveles del patrimonio.

j) Valora la importancia del patrimonio de manera holística y sistémica a partir, de su experiencia.

\section{Medios y materiales educativos}
a) Documento dossier didáctico.
b) Exposición teórica del docente (hace uso de pizarra, presentación power point).
c) Carpeta bibliográfica en formato digital (informes, tesis, libros, etcétera).
d) Papelógrafos y marcadores de tinta indeleble.
e) Historias diarias.
f) Guía de inventario patrimonial.

\section{Actividades}

Resolución de guía de inventario patrimonial de mi comunidad, análisis de cuestionario de preguntas y actividades lúdicas (carpeta bibliográfica digital, dossier didáctico, fuentes iconográficas (fotografías y recurso audiovisual).

\section{E. Procedimientos}

\section{Guía inventario patrimonial}

Trabajo individual

Pasos 
a) Lectura y primera impresión del dossier didáctico.

b) Ideas que se visualizan en el documento base (dossier), al igual aquellas procedentes de fuentes documentales y de internet.

\section{Trabajo en colectivo (grupal)}

\section{Pasos}

a) Sintetizar a partir de la experiencia en la comunidad de cada capacitando, los tipos y niveles de patrimonio tomando en cuenta su clasificación y tipo (natural, cultural e integral, tangible e intangible).

b) Discutir las ideas personales en torno a la justificación de dicho patrimonio, su importancia para la comunidad y las medidas para su protección/conservación.

c) Elaboración de síntesis haciendo uso de la guía patrimonial.

d) Socialización del trabajo final.

\section{Análisis de cuestionario de preguntas}

\section{Trabajo individual}

\section{Pasos}

a) Conocimiento y dominio del tema sobre las fiestas religiosas de mi comunidad.

b) Enlistar y enumerar de acuerdo a los meses del año las danzas que forman parte de dichas fiestas.

\section{Trabajo en colectivo (grupal)}

\section{Pasos}

a) Reflexionar sobre los aspectos sociales y espirituales en los que se basan dichas danzas.

b) Puesta en común de ideas junto a la presentación de una danza (baile) local.

\section{Actividades lúdicas para preescolar}

\section{Trabajo colectivo (grupal)}

\section{Pasos}

a) Formación de equipos de trabajo de acuerdo al área de conocimiento.

b) Proponer una actividad pedagógica (juego de representación de animales de la naturaleza) para ser trabajado al nivel de aula con los estudiantes de pre-escolar a partir, de las fuentes documentales e iconográficas.

c) Cada equipo deberá seleccionar como mínimo tres animales para representar, por lo que deberán de realizar la gestualidad propia de dichos animales. 
d) Por su parte el resto de los miembros del aula "adivinan" el tipo de animal.

\section{Trabajo complementario}

\section{Pasos}

a) Lectura sistemática del dossier didáctico para c/u de los encuentros de clases.

b) Búsqueda permanente de recursos pedagógicos para la elaboración del texto escolar con el eje transversal del patrimonio natural y cultural en los niveles de preescolar comunitario y formal, primaria regular y multigrado.

c) Acopio de las actividades trabajadas por c/u de los equipos en carpeta/portafolio.

\section{Unidad II: El patrimonio natural y cultural para las localidades}

\section{A. Objetivos}

a) Valorar la importancia que tiene el patrimonio natural y cultural en los territorios mayangna Sauni As y Sauni Arungka.

b) Difundir el patrimonio natural y cultural de los pueblos indígenas de la Costa Caribe como aporte para la valoración y el sostenimiento del patrimonio.

c) Reconocer el papel del turismo como una oportunidad para el desarrollo local.

d) Determinar la diferencia entre conservar y preservar.

e) Reconocer el papel ejercido del patrimonio en el desarrollo sustentable local.

B. Contenido conceptual, procedimental y actitudinal

a) Importancia del patrimonio natural y cultural para las localidades.

b) Patrimonio y pueblos indígenas.

c) Del recurso patrimonial a la oferta cultural: el caso del turismo como alternativa.

d) Qué es eso de conservar, diferencia entre conservar y preservar.

e) El patrimonio y el desarrollo sostenible.

f) Comprende la importancia del patrimonio natural y cultural para las generaciones futuras.

g) Analiza los conceptos y su interrelación desde su experiencia.

h) Identifica cada uno de los elementos que intervienen en el turístico como alternativa.

i) Interpreta los conceptos y su interrelación a fin de garantizar medidas de defensa y preservación del patrimonio natural y cultural.

j) Analiza e interpreta los conceptos y su interrelación desde su experiencia. 
k) Comprende el concepto de patrimonio natural y cultural y valora ambos como referente de la continuidad histórico-cultural de los pueblos.

\section{Medios y materiales educativos}
a) Documento dossier didáctico.
b) Exposición teórica del docente (hace uso de pizarra, presentación power point).
c) Carpeta bibliográfica en formato digital (informes, tesis, libros, etcétera).
d) Papelógrafos y marcadores de tinta indeleble.
e) Historias diarias.
f) Guía de inventario patrimonial.
g) Recurso audiovisual.

\section{Actividades}

Análisis de cuestionario de preguntas, resolución de guía de inventario del recurso patrimonial de mi comunidad con perspectivas de explotación turística y análisis de material audiovisual (documental patrimonial), tomando en cuenta la (carpeta bibliográfica digital, dossier didáctico, fuentes iconográficas procedentes de fotografías y de recurso audiovisual).

\section{E. Procedimiento}

Análisis de cuestionario y guía inventario patrimonial

\section{Trabajo individual}

\section{Pasos}

a) Formación de equipos de trabajo de acuerdo al área de conocimiento.

b) Discutir las ideas personales sobre los aspectos estudiados.

c) Sintetizar a partir, del uso de la guía patrimonial y aprovechamiento de los recursos turísticos locales.

d) Puesta en común (plenario).

\section{Análisis de material audiovisual (documental patrimonial)}

\section{Trabajo colectivo (grupal)}

\section{Pasos}

a) Conocimiento y dominio del tema.

b) Discutir las ideas personales sobre los aspectos estudiados.

c) Sintetizar a partir, del uso de la guía patrimonial y aprovechamiento de los recursos turísticos locales. 
d) Puesta en común (plenario).

\section{Trabajo complementario}

\section{Pasos}

a) Lectura sistemática del dossier didáctico para c/u de los encuentros de clases.

b) Acopio permanente de material escrito y gráfico en revistas, periódicos, tesis, informes y otros que servirán de insumo para la elaboración del texto escolar; todas estas especificaciones técnicas sobre el libro escolar serán entregados en un taller relacionado con técnicas de elaboración de textos escolares.

c) Acopio de las actividades trabajadas por c/u de los equipos en carpeta/portafolio.

\section{Unidad III: Gestión del patrimonio natural y cultural para las localidades}

\section{A. Objetivos}

a) Promover la valoración del patrimonio natural y cultural desde su reconocimiento hasta su gestión a través, de teorías, metodologías, herramientas y técnicas.

b) Brindar los instrumentos y herramientas metodológicas que permitan la gestión participativa, académica y de investigación en los bienes patrimoniales.

\section{B. Contenido conceptual, procedimental y actitudinal}

a) Gestión desde y para las localidades.

b) Planificación participativa en el contexto patrimonial.

c) Ventajas de la planificación participativa.

d) Conoce e internaliza la importancia de planificar, difundir y gestionar el patrimonio de manera holística y sistémica para las localidades.

e) Genera actitudes responsables hacia la necesidad de planificar, difundir, gestionar y apropiación de los bienes patrimoniales locales.

f) Activa el interés en otros ciudadanos (escuela, comunidad), para proteger su patrimonio local.

\section{Medios y materiales educativos}
a) Documento dossier didáctico.
b) Exposición teórica del docente (hace uso de pizarra, presentación power point).
c) Carpeta bibliográfica en formato digital (informes, tesis, libros, etcétera).
d) Papelógrafos y marcadores de tinta indeleble.
e) Historias diarias. 


\section{Actividades}

Análisis de cuestionario, en torno a la necesidad de planificar, difundir, gestionar y apropiación del patrimonio natural y cultural desde una perspectiva holística y sistémica para las localidades.

\section{E. Procedimientos}

Análisis de cuestionario

Trabajo colectivo (grupal)

Pasos

a) Formación de equipos de trabajo de acuerdo al área de conocimiento.

b) Discutir las ideas personales sobre aspectos como planificación, difusión, gestión integral del patrimonio natural y cultural que permitan definir rutas, circuitos turísticos y otros para su puesta en valor a nivel local y nacional.

c) Sintetizar a partir, del uso de la guía de cuestionario y socialización del mismo.

\section{Trabajo complementario}

\section{Pasos}

a) Lectura sistemática del dossier didáctico para c/u de los encuentros de clases.

b) Acopio permanente de material escrito y gráfico en revistas, periódicos, tesis, informes y otros que servirán de insumo para la elaboración del texto escolar; todas estas especificaciones técnicas sobre el libro deben de ser pensados para estudiantes de enseñanza pre-escolar y primaria y serán entregados en un taller relacionado con técnicas de elaboración de textos escolares.

c) Acopio de las actividades trabajadas por c/u de los equipos en carpeta/portafolio.

\section{Unidad IV: Derechos de los pueblos indígenas en el marco jurídico internacional-nacional}

\section{A. Objetivos}

a) Analizar las leyes nacionales y convenios internacionales que rigen la defensa y protección del patrimonio de manera íntegra.

b) Estudiar algunas experiencias desarrolladas e instrumentos técnico-legales sobre la protección del patrimonio natural y cultural.

\section{B. Contenido conceptual, procedimental y actitudinal}
a) Definiciones básicas sobre pueblos indígenas.
b) Conocimiento indígena y conocimiento occidental.
c) Principios de la nación territorial mayangna. 
d) Definiciones básicas de la ley general de educación y el SEAR.

e) La cultura en la constitución política de Nicaragua.

f) Ley 28, Estatuto de Autonomía de la Costa Caribe Nicaragüense.

g) Las leyes nacionales e internacionales para la conservación del patrimonio cultural y natural.

h) Interpreta los conceptos y su interrelación con el patrimonio desde su experiencia.

i) Reconoce la importancia del derecho consuetudinario de los pueblos indígenas.

j) Reconoce los principios y disposiciones contenidos en la ley general de educación al igual que el SEAR en torno a la Educación Intercultural Bilingüe.

k) Conoce e internaliza los artículos contemplados en la Constitución Política y el Estatuto de Autonomía relacionados a la cultura y derechos de los pueblos indígenas.

1) Subraya los procesos y sanciones que la Ley 1142 exige para la conservación y protección del patrimonio cultural.

m) Reconoce los procesos y sanciones establecidos en la Ley 217 relacionados al medio ambiente.

n) Reconoce los principios y disposiciones contempladas en el convenio internacional de salvaguardia del patrimonio cultural a nivel mundial.

o) Adquiere una actitud crítica frente a dogmas sociales, políticos y religiosos así, como defiende los valores históricos ancestrales que definen sus señas de identidad.

\section{Medios y materiales educativos}
a) Documento dossier didáctico.
b) Exposición teórica del docente (hace uso de pizarra, presentación power point).
c) Carpeta bibliográfica en formato digital (informes, tesis, libros, etcétera).
d) Papelógrafos y marcadores de tinta indeleble.
e) Historias diarias.

\section{Actividades}

Lectura crítica de postura sobre los principios y disposiciones de corte jurídico-legal que sustentan la protección y defensa del patrimonio natural y cultural de los pueblos indígenas de la Costa Caribe de Nicaragua.

\section{E. Procedimientos}

\section{Lectura crítica y analítica}

Trabajo colectivo (grupal) 


\section{Pasos}

a) Formación de equipos de trabajo de acuerdo al área de conocimiento.

b) Los capacitandos dispondrán de lecturas complementarias contempladas en el dossier didáctico relativo a ley de protección al patrimonio cultural de la nación o decreto de Ley 1142, los principios establecidos en la Ley 162 sobre el uso oficial de las lenguas en la Costa Caribe y los procesos y sanciones establecidos en la Ley 217 concerniente al medio ambiente.

c) Analizar y relacionar críticamente los documentos orientados.

d) La actividad debe ser entregada y se comentará en el aula de clases.

\section{Trabajo complementario}

\section{Pasos}

a) Lectura sistemática del dossier didáctico para c/u de los encuentros de clases.

b) Acopio de material escrito y gráfico en revistas, periódicos, tesis, informes y otros que servirán de insumo para la elaboración de la cartilla escolar.

c) Acopio de las actividades trabajadas por c/u de los equipos en carpeta/portafolio.

\section{La evaluación final del proceso enseñanza-aprendizaje}

La evaluación del módulo se realizó de forma continua a lo largo del mismo, teniendo en cuenta la evaluación de orientación y control que permitió medir el proceso de enseñanza-aprendizaje de los capacitados; pero, además, se hizo hincapié en la asistencia y participación. Conviene subrayar que a cada equipo de trabajo se les pidió que manejaran una carpeta/portafolio que funcionara como receptor y testigo de c/u de las actividades orientadas en el dossier; cada eje temático contempló actividades en forma colectiva e individual los que propiciaron la participación significativa de los capacitados y estuvieron orientados a desarrollar habilidades y actitudes coherentes de acuerdo a los propósitos del diplomado y que en general sumaron un valor porcentual de (80\%). No obstante, el producto evaluado de mayor significación y ponderación del módulo fue la realización de un informe final (20\%) que tuviera una aplicación directa con respecto al proceso de aprendizaje de las unidades temáticas del curso por lo que los criterios tomados en cuenta en la evaluación de éste fueron creatividad, calidad en la presentación de ideas con claridad y precisión del documento, la utilización de fuentes y bibliografía además, del uso de elementos o recursos iconográficos que servirían de insumo para la elaboración posterior de los textos escolares.

\section{Relevancia del dossier didáctico como apoyo a la docencia de la EIB}

En definitiva, el impacto del módulo didáctico no solo representa un aporte al sistema de Educación Intercultural Bilingüe (EIB), por el contrario, la elaboración participativa del currículo o bien la adaptación curricular como sucede en este caso de estudio que supone la (...) profundización (...) de los contenidos curriculares (Picardo, 2004, p. 19), es un procedimiento que permite avanzar hacia la meta, al señalar que la educación intercultural debe ser un asunto de interés para toda la ciudadanía. En efecto, los lineamientos curriculares son resultado de la búsqueda realizada conjuntamente por docentes y asesores pedagógicos de educación básica y media del MINED, en la elaboración, desarrollo y adaptación del currículo por lo que el patrimonio natural y cultural del pueblo mayangna es una de las manifestaciones fundamentales de su cultura y como tal debe ser incluido en los distintos niveles educativos. El docente, además, de identificar y valorar el patrimonio natural y cultural comprenderá la diversidad del patrimonio de manera holística manifestado en su idioma, conocimiento ancestral y expresiones artísticas todo lo anterior como eje vertebrador de los derechos humanos, sociales, económicos y culturales del pueblo panamahka. 
En consecuencia, algunas evidencias que permiten hacer una evaluación positiva sobre el impacto que tuvo el desarrollo del módulo está al retomar el uso de los materiales didácticos en el que es posible constatar que al menos un $93 \%$ de los capacitados reconocieron que el material entregado (dossier), fue útil, adecuado y claro, acorde a los objetivos y contenidos del módulo y como apoyo efectivo en el proceso de enseñanza-aprendizaje del curso impartido; (el dossier fue entregado además, en formato digital de manera que pueda ser replicado a docentes, estudiantes y personas afines al área de conocimiento). Un elemento que respalda lo anterior es al analizar el cumplimiento de la metodología planificada la que se caracterizó por ser motivadora y aplicar distintas estrategias didácticas que fomentaron el trabajo grupal y colaborativo; pero, además, reconocer el enorme interés mostrado por parte de los capacitados con sus participaciones sistemáticas que fueron sustanciales para el proceso de enseñanza. Al revisar el rendimiento académico en general el promedio global del grupo alcanzó un 91.3\%, con calificación máxima de 96 y mínima de 80.

Por otro lado, expresar mi gratitud por haber tenido la oportunidad de haber trabajado con un excelente grupo de compañeras y compañeros de singular tenacidad y motivación de superación que en cualquier círculo de trabajo es meritorio reconocerlo ya que sus experiencias, intereses y expectativas desde la labor que ejercen fueron enriquecedoras para generar y mediar el conocimiento colectivo en el diplomado. Finalmente, los docentes panamahka de los territorios Sauni As y Sauni Arungka tienen un reto por delante ya que el patrimonio natural y cultural se acrecienta y se defiende mejor cuanto más lo aprecian las personas que conviven con él.

\section{Lista de referencias}

Álvarez, M. (2017). Costa Caribe de Nicaragua: autonomía, interculturalidad, ética y emancipación social. Recuperado de revistashumanidadescj.unan.edu.ni/index.php/Humanismo/article/view/198

Conzemius, E. (2004). Estudio Etnográfico sobre los miskitos y sumos de Honduras y Nicaragua. Colección cultural centroamericana, serie etnológica, $\mathrm{n}^{\mathrm{O}} 21^{\mathrm{a}}$ edición, Managua, Fundación vida.

González Hodgson, K. (2017). Patrimonio Integral de la Costa Caribe de Nicaragua. (Módulo II). UNAN-Managua/FASOC.

Houwald, Gotz Von. (1984). Tradiciones Orales de los Indios Sumus. Estudios americanistas de Bonn, República Federal de Alemania, Bonn.

Houwald, Gotz, Von. (2003). Mayangna, Apuntes sobre la Historia de los Indígenas Sumus en Centroamérica. Managua: Fundación Vida, 2003. pp 668.

Hurtado, de Mendoza, L. (2000). Identidad Cultural Mayangna en Nicaragua. Editorial Sociedad y Ambiente. Managua.

La Constitución Política de Nicaragua y sus reformas. Corte Suprema de Justicia. Publicada en la Gaceta, Diario Oficial \# 05 del o9 de enero de 1987. Recuperado de: http://www.poderjudicial.gob.ni/pjupload/archivos/documentos/LA_CONSTITUCION_POLITICA_Y_SUS_REFORMAS(3).pdf

Ley $\mathrm{N}^{\circ}$ 28, Estatuto de Autonomía de las Regiones de la Costa Atlántica de Nicaragua, publicada en La Gaceta, Diario oficial, \# 238 del 30 de Octubre de 1987.

Ley $\mathrm{N}^{\circ}$ 162, Ley de Uso Oficial de las Lenguas de las Comunidades de la Costa Atlántica de Nicaragua, publicada en La Gaceta, Diario Oficial, \# 183 del 27 de Septiembre de 1996. 
Ministerio de Educación (MINED, 2009). “Currículo Nacional Básico”. Diseño Curricular del Subsistema de la Educación Básica y Media Nicaragüense.

Ortiz, A. (2017). Marco Legal de los Pueblos Indígenas. Taller de Inducción impartido en el marco del Proyecto Generando Capacidades en Docentes y Materiales Didácticos para el Fortalecimiento del Programa de Educación Intercultural Bilingüe (EIB). (Inédito). Bonanza, 7 y 8 de julio.

Picardo, O. (2004). Diccionario Enciclopédico de Ciencias de la Educación. 1 $^{\mathrm{a}}$ edición, San Salvador, El Salvador, C.A: Centro de Investigación Educativa, Colegio García Flamenco. pp. 400. 